\title{
Gas phase hydrodynamics in a surface-aerated tank with a long-short blades agitator
}

\author{
Yongjun $\mathrm{Wu}^{1}$, Jian Wang ${ }^{2}$, Pan You ${ }^{1}$, and Peicheng Luo ${ }^{1}$ \\ ${ }^{1}$ Southeast University \\ ${ }^{2}$ Beijing Institute of System Engineering
}

September 25, 2021

\begin{abstract}
This work aims to study the gas phase hydrodynamics in a stirred tank with a surface-aerated long-short blades agitator by the Eulerian-Eulerian approach coupled with population balance model. Predicted local gas holdup and bubble size distribution agree well with those measured by a conductivity probe technique. The predictions demonstrate that the pressure depression in the center is the main driving force for gas suction and the downward flow carries the bubbles down to redistribute in the whole tank. The gas phase has higher gas holdup with large bubble size in the upper part and lower gas holdup but with small bubble size in the lower part of the tank. The predicted gas-liquid mass transfer coefficients agree well with our previous experimental results and just depends on the power consumption per unit volume when the aspect ratio of the liquid height to the tank diameter varies from 1.1 to 2.0 .
\end{abstract}

\section{Hosted file}

20210830_surface_aerator_LSB.docx available at https://authorea.com/users/437253/articles/ 539018-gas-phase-hydrodynamics-in-a-surface-aerated-tank-with-a-long-short-bladesagitator

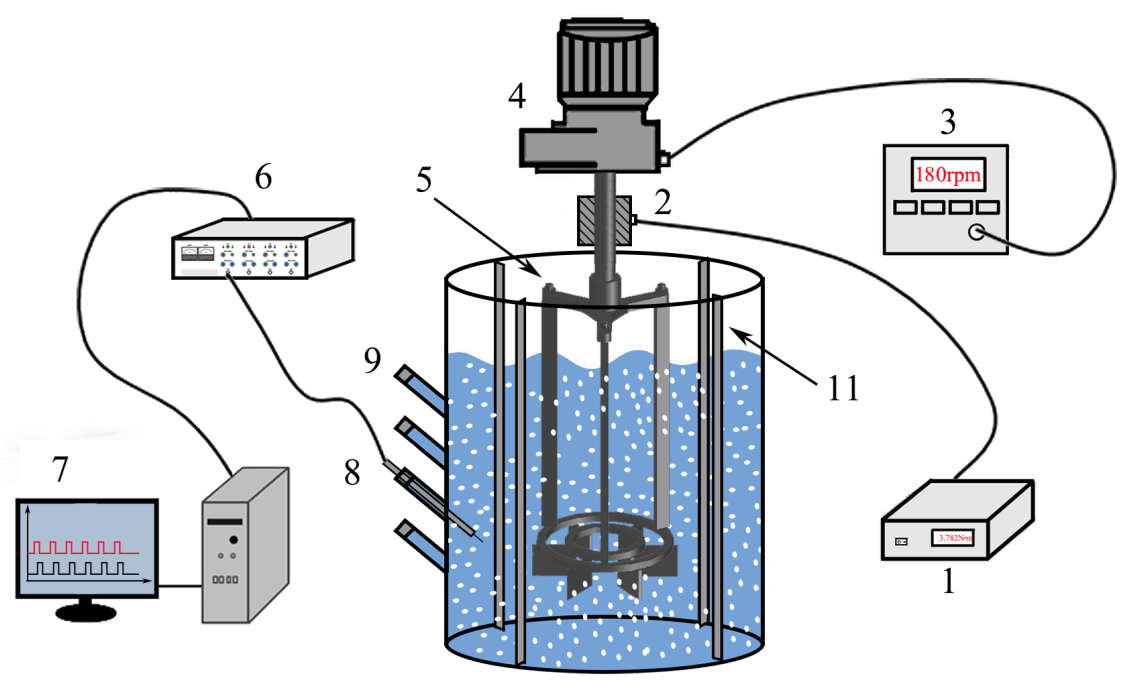

10 


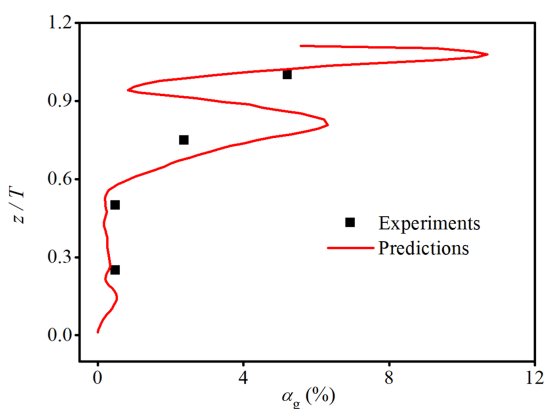

(a)

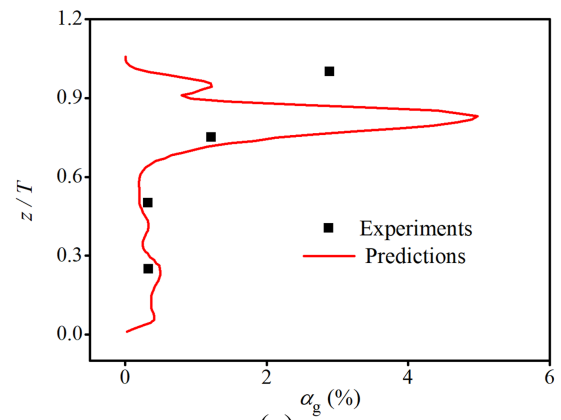

(c)

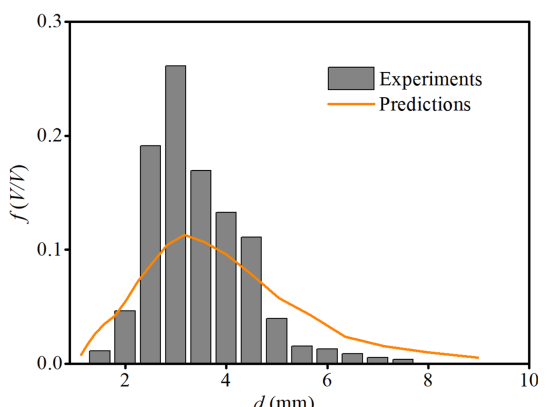

(a)

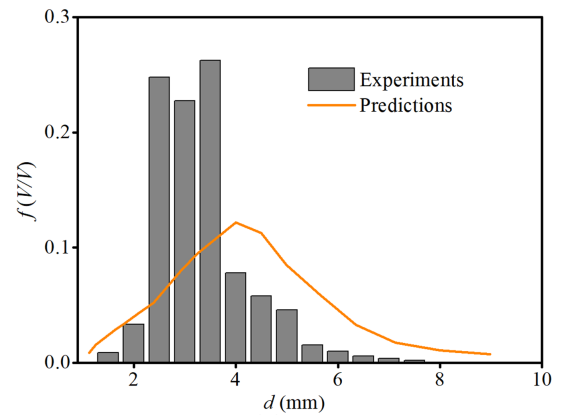

(c)

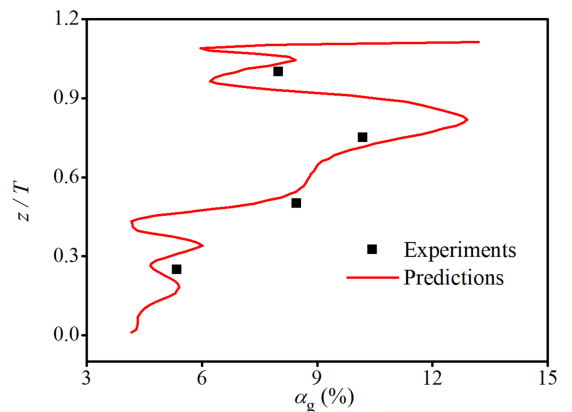

(b)

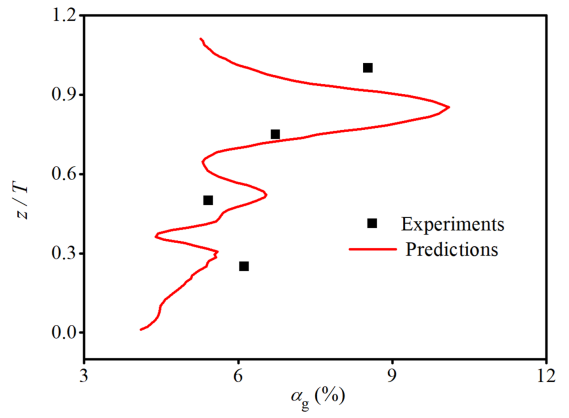

(d)

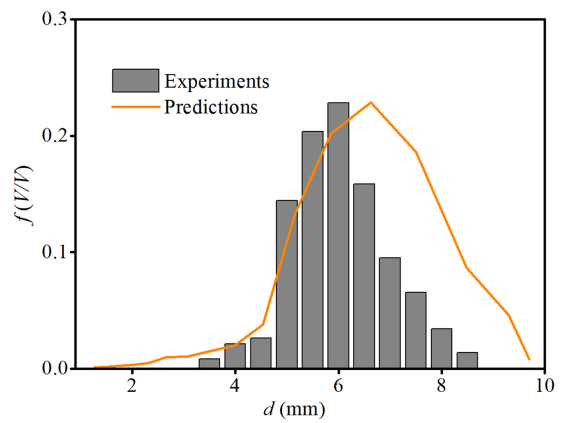

(b)

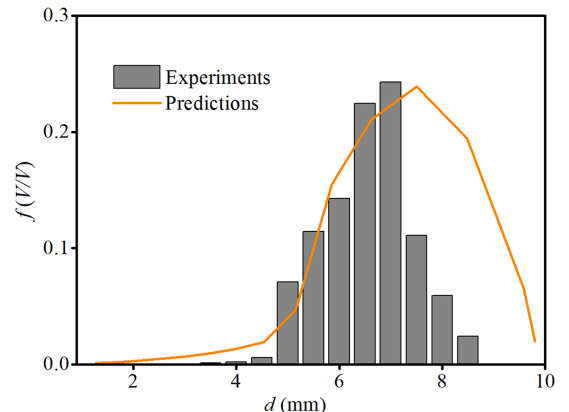

(d) 


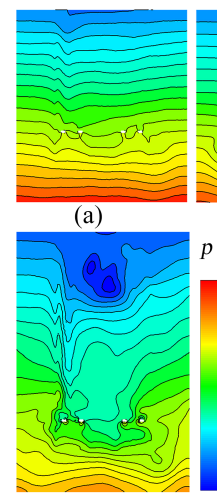

(d)
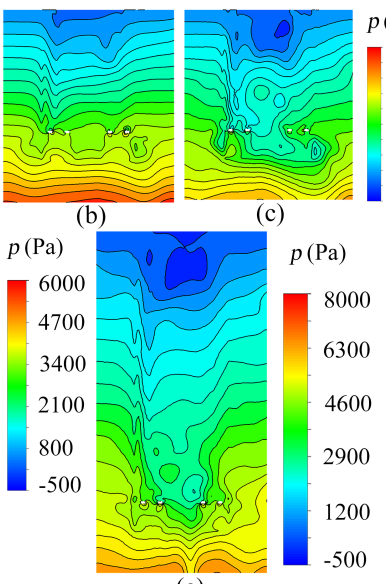
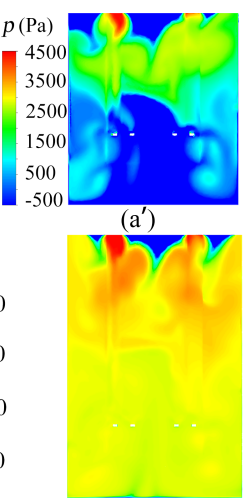

(d')

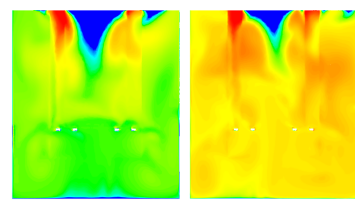

(c')

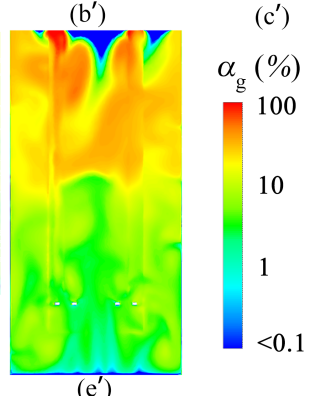

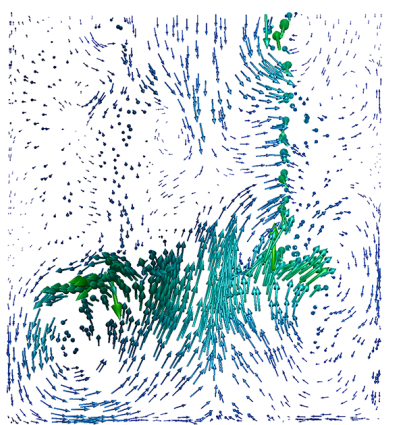

(a)

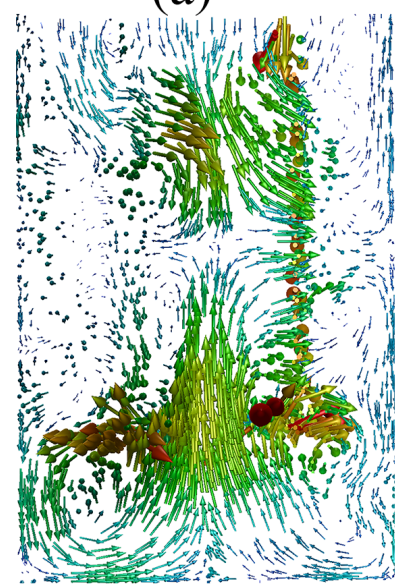

(d)

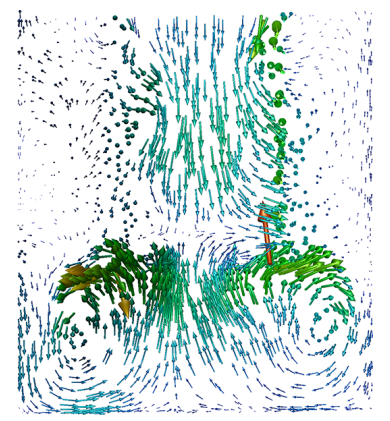

(b)

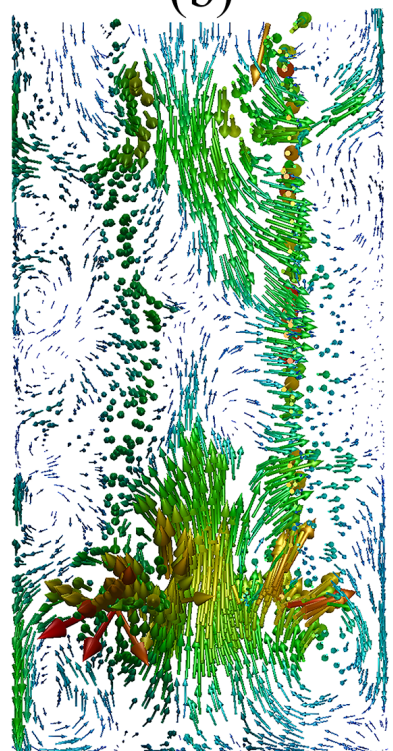

(e)

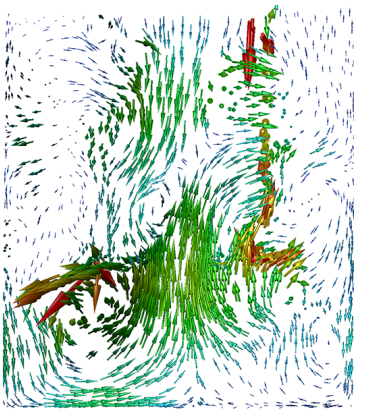

(c)

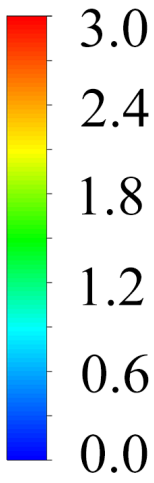




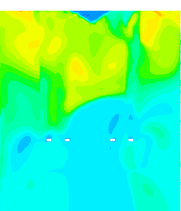

(a)

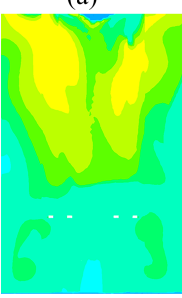

(d)

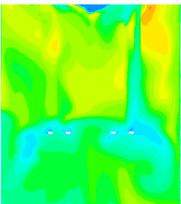

(b)

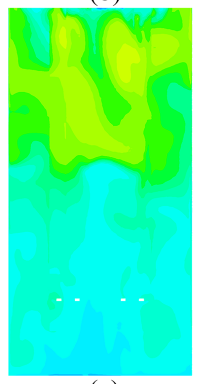

(e)

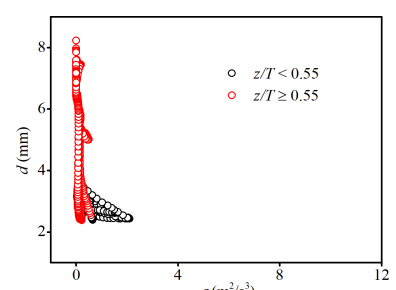

(a)

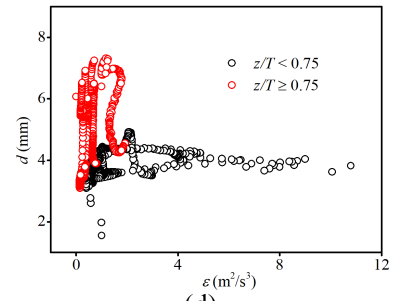

(d)

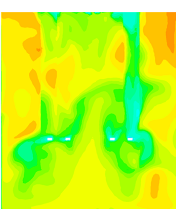

(c)

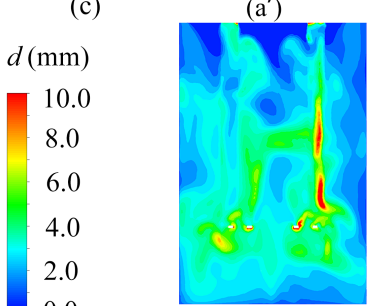

(d')

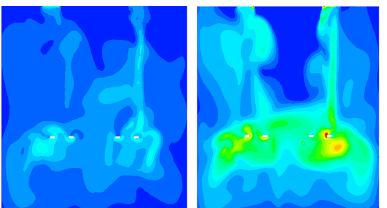

$\left(b^{\prime}\right)$

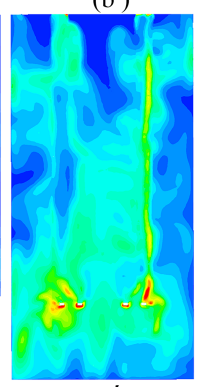

(e')

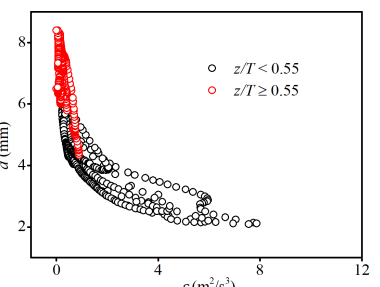

(b)

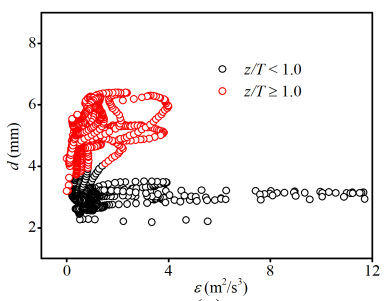

(e)

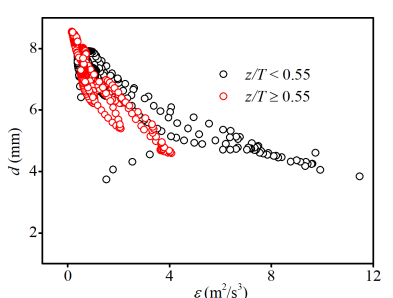

(c)

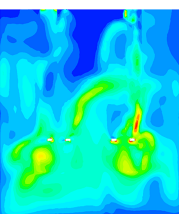

$k\left(\mathrm{~m}^{2} / \mathrm{s}^{2}\right)$

0.5

0.4

0.3

0.2

0.1

0.0 


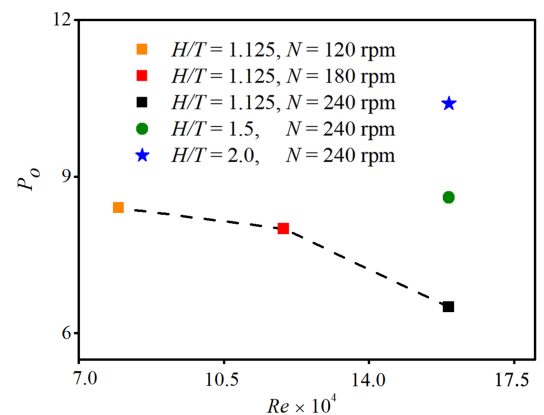

(a)

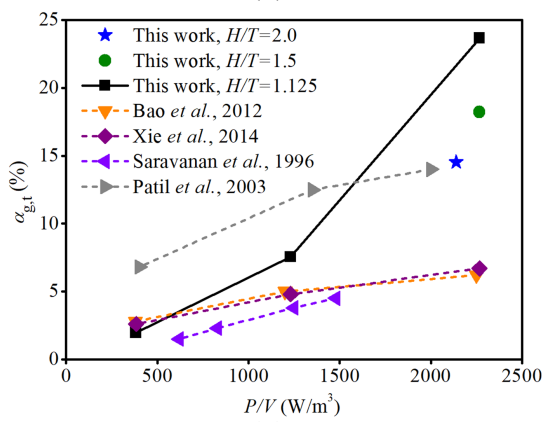

(c)

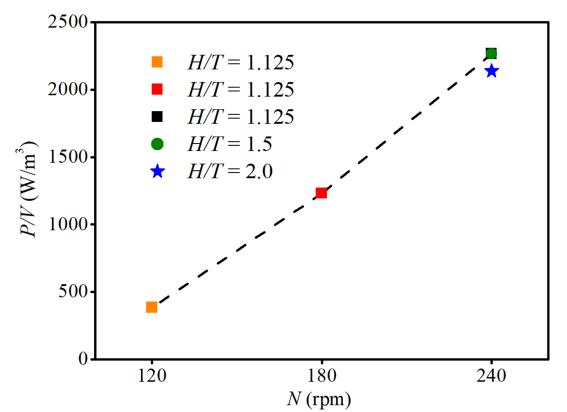

(b)

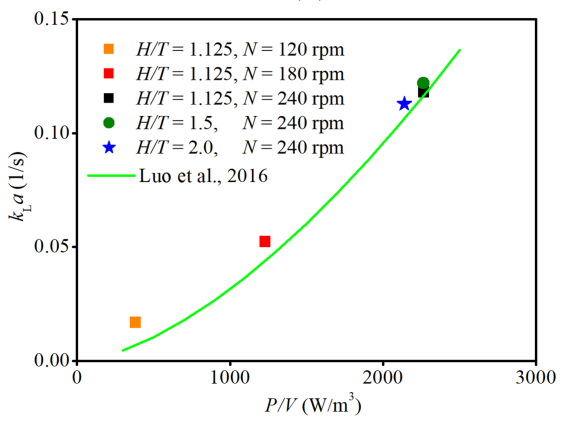

(d)

\section{Hosted file}

Table.docx available at https://authorea.com/users/437253/articles/539018-gas-phasehydrodynamics-in-a-surface-aerated-tank-with-a-long-short-blades-agitator 